\title{
Impacts Sociaux Et Environnementaux De L'orpaillage Sur Les Populations De La Region Du Bounkani (Cote D'ivoire)
}

\author{
AFFESSI Adon Simon \\ Université PELEFORO Gon Coulibaly (Korhogo- Côte d'Ivoire) \\ KOFFI Koffi Gnamien Jean-Claude \\ Université Félix HOUPHOUËT-BOIGNY (Abidjan - Côte d'Ivoire) \\ SANGARE Moussa \\ Université PELEFORO Gon Coulibaly (Korhogo- Côte d'Ivoire) \\ Membres du Laboratoire d'Etudes et de Recherches Interdisciplinaires en \\ Sciences Sociales(LERISS) \\ Université Félix Houphouët-Boigny d'Abidjan
}

doi: 10.19044/esj.2016.v12n26p288 URL:http://dx.doi.org/10.19044/esj.2016.v12n26p288

\begin{abstract}
This work highlights the effects of the practice of rural panning. This activity is considered a factor in promoting better condition of life of the miners. Its implementation modifies the relations of individuals with nature. The action of the miners, in their quest for wellness, causes social, economic and relationship with adverse environmental consequences. The study method was to collect information from resource persons and the use of functionalist theories and structural-functionalist. The results indicate that gold mining activity, although revenue generating negatively affect the environment, food safety and the health of populations in the Region Bounkani.
\end{abstract}

Keywords: Gold panning-social changes- environment- exploitation

\section{Résumé}

Le présent travail met en évidence les effets de la pratique de l'orpaillage en milieu rural. Cette activité est considérée comme un élément favorisant une meilleure condition d'existence des orpailleurs. Sa mise en œuvre modifie les rapports des individus avec le milieu naturel. L'action des orpailleurs, dans leur quête de bien-être, provoque des changements sociaux, économiques et relationnels avec des conséquences environnementales néfastes. La méthode d'étude a consisté à recueillir des informations auprès de personnes ressources et au recours aux théories fonctionnaliste et structurofonctionnaliste. Les résultats obtenus indiquent que l'activité d'orpaillage, 
bien que génératrice de revenus, affecte négativement l'environnement, la sécurité alimentaire et la santé des populations dans la Région du Bounkani.

Mots clés: Orpaillage-changements sociaux-environnement-exploitation

\section{Introduction}

La dégradation de l'environnement demeure aujourd'hui l'une des préoccupations fondamentales des politiques gouvernementales à travers le monde. De Rio de Janeiro en 1992 à Johannesburg en 2002, la question environnementale a suscité beaucoup de réflexions et de recommandations relatives à sa conservation, car la planète n'a jamais connu un appauvrissement écologique d'une telle ampleur. Nombreux sont aussi les facteurs qui, de manière plus ou moins insidieuse participent à la dégradation de l'environnement : pollution des sols, des eaux et de l'air, pratiques agricoles et forestières et ces dernières années l'exploitation artisanale de l'or (Nations Unies, 2002).

L'extraction artisanale de l'or constitue une réalité incontournable en milieu rural, une occupation au même titre que l'agriculture et l'élevage (Ndela, 2008). C'est un moyen efficace de lutte contre la pauvreté. Cependant, cette activité minière artisanale comporte des inconvénients notamment sur les ressources naturelles, la santé humaine mais également au plan social (Jaques et al, 2004). Sur le plan social et sanitaire, sur les sites d'orpaillage de Siguinvoussé, Pouskgin et Touwaka du Burkina-Faso, les populations sont exposées à un risque de maladies infectieuses liées au manque d'installations sanitaires appropriées (Jaques et al, idem.). Les conditions d'hygiène, d'alimentation et les comportements à risques sont la cause de la détérioration de la santé des orpailleurs. Les populations des sites d'orpaillage sont souvent confrontées au paludisme, aux maladies diarrhéiques, aux infections respiratoires aigües et aux IST/VIH-SIDA. Au plan environnemental, les eaux de consommation de même que l'air sont pollués par la présence de l'activité d'orpaillage. Le constat est que l'exploitation artisanale de l'or est à l'origine de plusieurs désagréments au niveau de l'environnement, de la santé des acteurs et de la sécurité sociale. Ce qui démontre les effets néfastes des extractions minières sur l'environnement (Orcade, 2006), bien que l'orpaillage contribue au développement socio-économique.

Concernant la Côte d'Ivoire, l'orpaillage est une activité très ancienne bien que non reconnue officiellement. Elle demeure dans certaines régions une source de revenus et d'emplois malgré les menaces qu'elle fait peser sur l'environnement (Kouadio, 2003). Ce secteur minier contribue à hauteur de 2,3\% du PIB (dont 0,7\% pour l'or) en 2012 et emploie environ 30000 personnes. Les investissements pour la même année se chiffrent à plus de 103,8 milliards de F CFA (MMEP, 2013). D'après le Ministère ivoirien des 
Mines et de l'Energie, le pays regorge plus de 1.000 sites d'exploitation artisanale de l'or et 500.000 personnes vivent de cette activité en milieu rural.

Dans la région du Bounkani, l'activité aurifère est exercée. Elle constitue une source de revenus pour un nombre important des populations et favorise la réalisation de plusieurs infrastructures dont des établissements scolaires, des habitations, etc. Les méthodes et techniques d'exploitation étant très traditionnelles pour les populations de cette région, on assiste de plus en plus à une prolifération des sites. L'exploitation aurifère engendre des effets néfastes sur la santé des orpailleurs ainsi que sur celles des populations riveraines, sur l'agriculture et sur l'environnement. L'intérêt de cette étude est de montrer aussi, comme c'est le cas ailleurs dans certains pays africains (Burkina Fasso, Mali...) qui pratiquent l'activité d'orpaillage, les nuisances d'une activité considérée comme génératrice d'emplois et de revenus à travers ses techniques de mise en œuvre et les conditions de création. Alors, quels sont les impacts des exploitations artisanales de l'or sur l'environnement et l'organisation socioéconomique des populations?

De cette question centrale se dégage des questions subsidiaires : quels sont les problèmes environnementaux provoqués par les méthodes et techniques de l'exploitation traditionnelle de l'or ? Comment améliorer la performance environnementale de l'orpaillage tout en la maintenant?

Cette étude vise à identifier les impacts environnementaux et sociaux de l'orpaillage en vue d'améliorer la performance environnementale et les conditions socio-économiques des orpailleurs de la Région du Bounkani.

\section{Methodologie}

La méthodologie a pour axe de référence scientifique le développement rural appliqué au changement social. C'est par rapport à cet axe que l'enquête qualitative a été privilégiée, fondée sur la logique de la dynamique du changement social. Cette dernière a été motivé par la prise en considération de tous les acteurs sociaux impliqués dans le projet de développement rural dans un souci de représentativité qualitative et surtout par la nature des variables essentielles mises en relation et dont le contrôle ne nécessite pas de données chiffrées. Le champ social consiste en l'identification des unités sociologiques ou populations cibles d'enquête. Ainsi, l'identification des impacts environnementaux a-t-elle été faite à partir des entretiens avec le personnel des Directions Départementales de la Société pour le Développement Minier de la Côte d'Ivoire (SODEMI), des eaux et forêts et les exploitants artisanaux, c'est-à-dire les orpailleurs, les négociants et les populations riveraines où se déroule l'activité d'exploitation de l'or.

Pour répondre aux questions qui sous-tendent ce travail de recherche, la méthode fonctionnaliste a été retenue pour interpréter les données recueillies par entretiens. Les méthodes d'extraction de l'or utilisées ainsi que 
les procédés et la manière d'agencer les activités sont à la base des problèmes de santé auxquels les travailleurs ainsi que les riverains sont confrontés. A ce niveau, le structuro-fonctionnalisme permet d'appréhender les structures et leur fonctionnement afin de comprendre et d'expliquer les différents problèmes sociaux et environnementaux. En effet, l'exploitation artisanale de l'or en tant que fait socioéconomique ne peut pas se comprendre de façon isolée. La méthode fonctionnaliste permet de ressortir les différents rôles des acteurs ou intervenants de cette activité et les différentes fonctions de toutes les autres activités en relation avec l'orpaillage. Les pratiques aurifères dans la région du Bounkani s'intègrent dans un cadre d'activité réglementé dans lequel les acteurs mettent en place une dynamique fonctionnelle pour atteindre des buts communs. L'exploitation artisanale à première vue parait inorganisée ; pourtant, elle obéit à un certain nombre de règles à l'instar des industries aurifères : les exploitations de type industriel et artisanal ont chacun un système d'organisation structurel et fonctionnel. Par conséquent, l'approche structuro-fonctionnaliste telle que définie par Parsons offre un cadre d'analyse afin d'appréhender les systèmes d'organisation et les pratiques mises en place pour atteindre les buts respectifs.

\section{Resultats}

\section{Chapitre $\mathbf{i}$ : caracterisation des acteurs et etapes d'extraction de l'or Les détenteurs des sites}

Ce sont des individus qui possèdent des ressources plus ou moins importantes permettant de financer les activités de prospection. C'est à eux qu'appartiennent les sites.

\section{Les orpailleurs}

Ils sont principalement les personnes dont l'activité permet dans une certaine mesure l'exploitation de l'or. Parmi eux, on peut citer les propriétaires des puits, les creuseurs, les concasseurs et les laveurs.

Le propriétaire d'un puits peut être tout individu, femme ou homme, qui dispose d'un fond de roulement suffisant pour financer l'activité d'exploitation de l'or. C'est lui qui recrute le personnel d'exploitation et qui prend en charge toutes les activités y compris la nourriture et les besoins de son équipe jusqu'à l'abandon du puits.

Le travail du personnel d'exploitation consiste à creuser la roche, à la transférer en dehors du puits et à la transporter sur les aires de concassage. Au niveau du concassage, ce sont les personnes de la même équipe, aidées par des journaliers, femmes et hommes qui assurent le concassage des roches extraites des puits. Ce sont ces mêmes personnes qui assurent le lavage de la roche après concassage et broyage. Les broyeurs sont des personnes qui disposent de meules qu'ils utilisent pour broyer la roche après concassage. 


\section{Les commerçants}

Tous les corps de métiers rencontrés en ville se retrouvent sur les sites d'orpaillage. C'est ainsi qu'on trouve des femmes et des hommes travaillant comme tenanciers des restaurants, boutiquiers, vendeuses de légumes et fruits, etc., afin de nourrir la communauté minière.

\section{Les différentes étapes de l'extraction artisanale de l'or sur les sites}

Sur les sites d'orpaillage, le lieu de creusage du minerai est appelé « site » et le lieu de concassage à l'extraction est appelé " comptoir ». Le comptoir est également le lieu de vente de l'or.

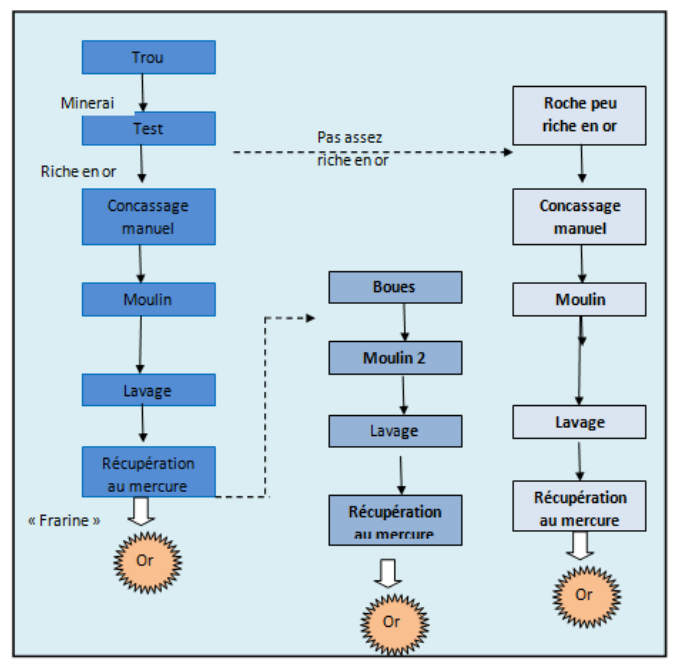

Figure 1: Etapes d'extraction de l'or rencontrées sur les sites

\section{Identification du site ou prospection}

L'identification du site est la toute première phase de l'extraction de l'or. Dans les mines artisanales, elle est faite par des prospecteurs. La recherche se fait le plus souvent par des observations de la zone par des anciens (orpailleurs expérimentés). Elle consiste à trouver un indicateur attestant de la présence de l'or sur le site. Après la découverte de cet indicateur, un test d'extraction est fait sur une profondeur de $0.5-1 \mathrm{~m}$ pour avoir une idée de la quantité d'or qui peut être présente. Cette quantité détermine si la zone est exploitable ou non. La prospection se fait avec des outils tels que la pioche et la pelle pour creuser, voire déblayer la roche. Suivant l'orientation du filon (prolongement de la roche qui contient l'or) des trous ou puits sont faits constituant une ligne d'orpaillage. 


\section{Fonçage}

Le fonçage consiste à creuser des trous afin d'atteindre le minerai qui se trouve dans une roche. Il se fait manuellement et constitue la phase la plus difficile et pénible du circuit d'extraction. L'orientation du trou suit le lit du minerai ; elle peut être verticale ou horizontale. Les trous sont rectangulaires de dimensions variables $1,5 \times 1 \mathrm{~m}$ et $3 \times 2 \mathrm{~m}$, la profondeur varie de 4 à $20 \mathrm{~m}$ voire $24 \mathrm{~m}$ en fonction de la richesse du puits.

Pour éviter des éboulements et consolider les parois des trous, des soutènements sont faits avec des troncs d'arbres. Le soutènement se fait au fur et à mesure qu'avance le creusage compte tenu de la faible portance du sol. Le soutènement utilise en moyenne 20 bois pour un mètre de profondeur. Le travail se fait en équipe de dix à onze personnes par trou travaillant jour et nuit en se relayant. Le matériel de fonçage est constitué de pelles, pioches, marteaux, pics, sacs, cordes et machettes. Les torches à piles sont également utilisées pour éclairer les creuseurs à l'intérieur du trou. Les dynamites sont très peu employées sur le site du fait de la faible résistance du sol (argileux). Le fonçage d'un trou est arrêté une fois que la nappe phréatique est atteinte et que le propriétaire du trou ne dispose plus de pompe suffisamment puissante pour dénoyer le puits et l'évacuer à la surface. Le minerai extrait est acheminé à l'extérieur dans des sacs et transporté au niveau du poste de stockage à l'aide des charrettes.

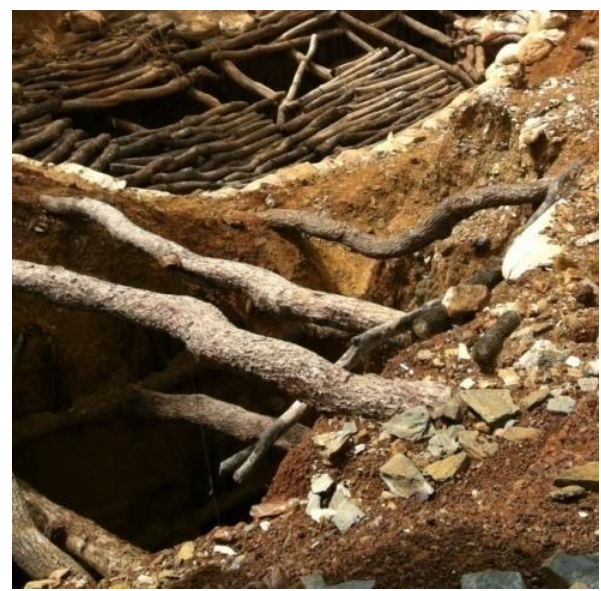

Photo 1: Fonçage d'un puits

\section{Test du minerai}

Afin de vérifier si le minerai extrait contient de l'or, un test est fait au niveau du site. Si le test est positif, on récupère le minerai pour la suite de la chaine ; dans le cas contraire, il est mis en dépôt. Ce test consiste à broyer la roche avec un mortier et un pilon en métal, ensuite une assiette et une cuvette permettent de laver le minerai et évaluer sa teneur en or. 


\section{Concassage}

Cette phase consiste à rendre la taille du minerai extrait en petite taille. Il se fait sur l'air de concassages. Le concassage est manuel et se fait à l'aide d'un marteau, d'une enclume (pierre de granite), d'un nœud de sac pour éviter les projections de particules et protéger les doigts. Le plus souvent, le concassage est fait par des jeunes ou toute autre personne à 1000 FCFA le sac de 50kg ou par le propriétaire du sac s'il n'est pas fatigué.

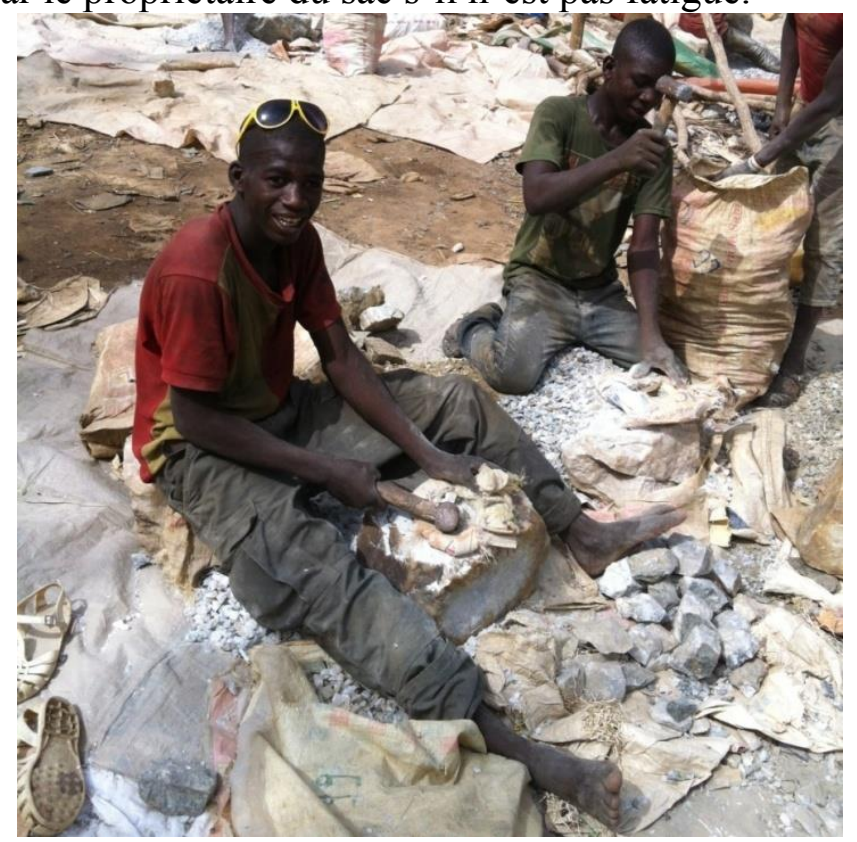

Photo 2: Concassage du minerai

\section{Broyage}

Durant cette phase le minerai est réduit en poudre, communément appelé farine. Le minerai est broyé dans les moulins à énergie gazole deux fois de suite et est séché entre chaque broyage quand le taux d'humidité est élevé. Le broyage est fait par deux à trois personnes dans un moulin. Au total, quatre moulins sont actifs sur le site de Lomidouo et cinq sur le site de Kintan. Le dispositif de broyage est constitué d'un moulin, d'un moteur relié au moulin par une courroie pour le faire fonctionner, deux fûts de gasoil pour alimenter le moteur, deux fûts d'eau pour refroidir le moteur, d'un "dynamo à meule » qui sert à aiguiser les meules, des cuvettes pour recueillir la farine, les bâches pour sécher la farine. 


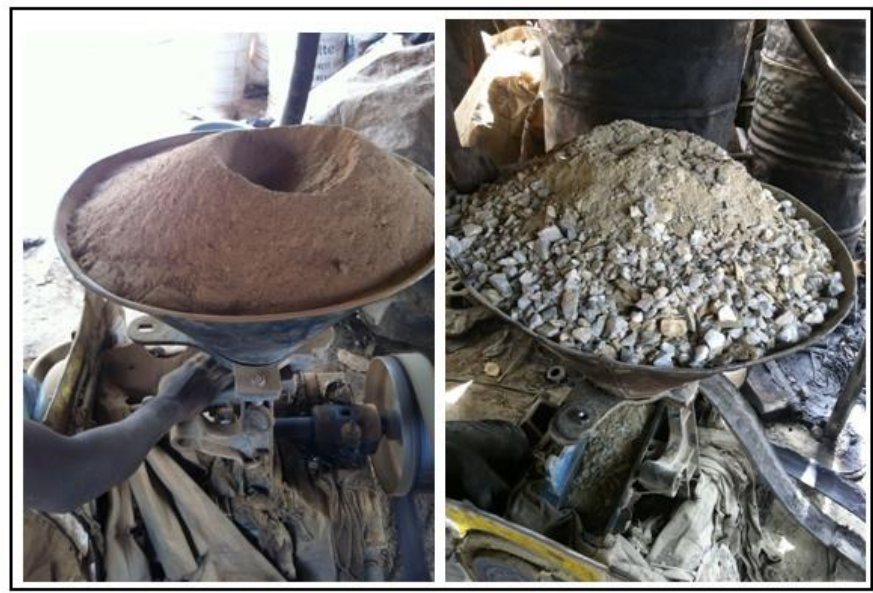

Photo 3: Broyage du minerai

\section{Le lavage}

La farine issue du broyage ( $\mathrm{sac}$ de $50 \mathrm{~kg}$ ) est mélangée avec de l'eau. Ce mélange est ensuite lavé sur une rampe. Une petite quantité du mélange est placée dans une passoire, ensuite de l'eau y est versée pour être liquéfié. La rampe est couverte d'un tapis (moquette) qui, par gravimétrie, piège l'or et la matière légère est entrainée vers le bas dans un trou peu profond. Le tapis est rincé dans une cuvette d'eau pour être débarrassé de l'or. Cette opération se fait après une certaine quantité de mélange lavé. Après cette étape, la boue restée dans la cuvette où est rincé le tapis est nettoyée jusqu'à obtention de la « poudre noire ». Cette opération qui se déroule au niveau du comptoir est surveillée par environ 25 personnes qui sont employés par le propriétaire du site. Leur rôle consiste à surveiller les orpailleurs afin que ceux-ci ne détournent pas le minerai au cours de cette opération.

Après ce lavage, la matière légère récupérée dans le trou est laissée à la décantation. Après décantation, les boues sont récupérées par les femmes pour être traitées de nouveau selon le même processus qu'initialement. 


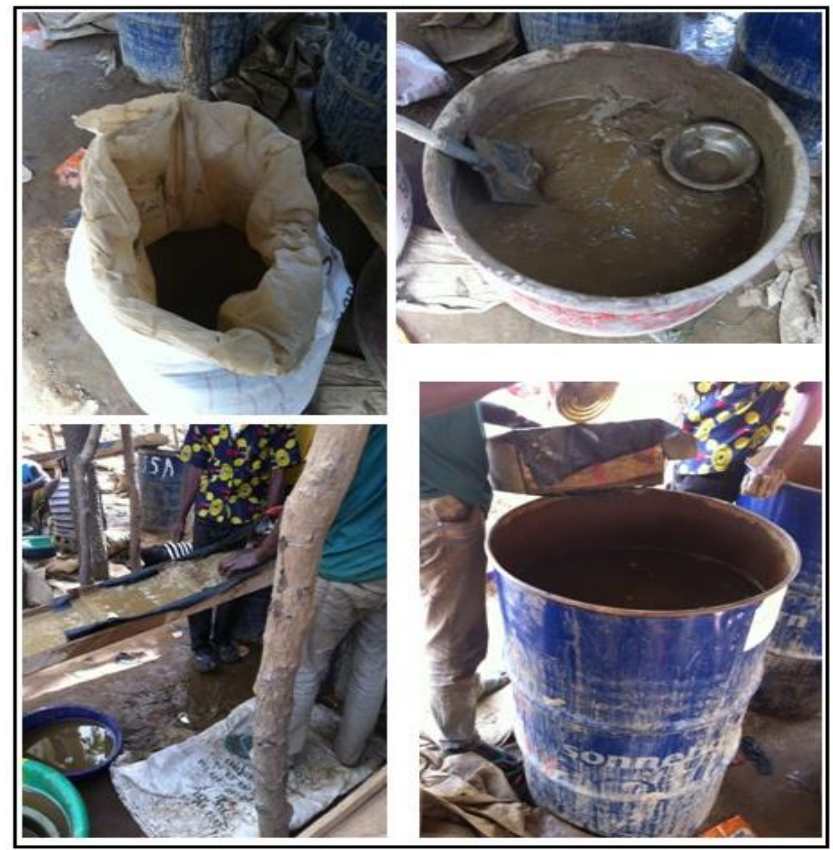

Photo 4: Les opérations de lavage au comptoir

\section{Récupération de l'or}

C'est la dernière phase d'extraction de l'or. Les orpailleurs de cette zone utilisent un moyen, à savoir l'amalgamation au mercure $(\mathrm{Hg})$. La poudre noire obtenue après le lavage est mélangée à mains nues au mercure pour amalgamation. L'ensemble or-mercure sera ensuite brûlé au chalumeau pour avoir l'or. L'or est pesé sur place par un acheteur qui est un employé du propriétaire du site.

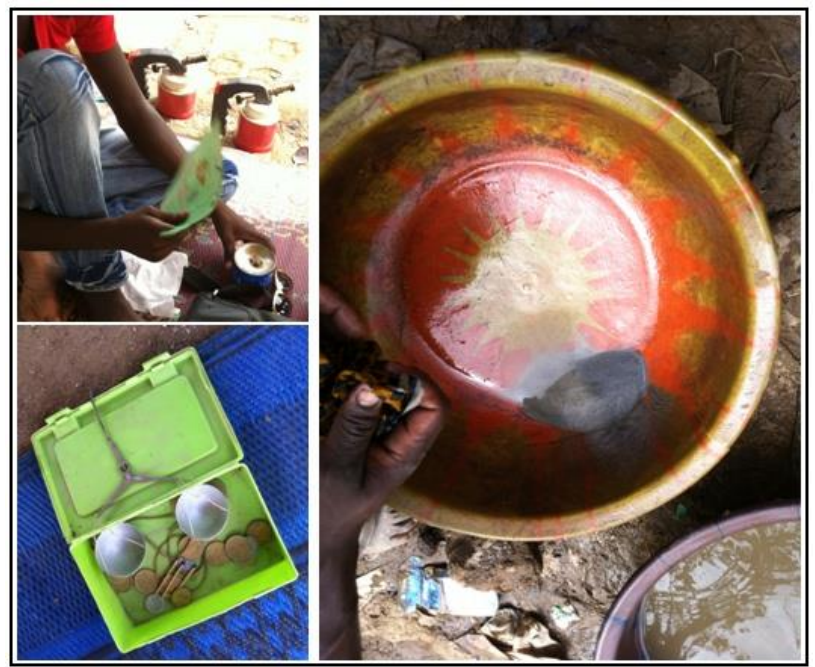

Photo 5: Récupération au mercure, agglutination et pesée de l'or au niveau du comptoir 


\section{Chapitre II. Impacts De L'activite Aurifere}

\section{Impact sur la faune}

Les activités d'orpaillage sur les sites de Lomidouo et Kintan ont contribué à la perte des espèces fauniques de cet espace dû à la déforestation et à la production sonore. L'orpaillage utilise beaucoup de bois lors du fonçage pour le soutènement des parois des puits. Dès lors, cette activité entraine la destruction de niches écologiques et la diminution de certains animaux. Les activités ayant un impact direct sur la faune sont essentiellement le fonçage et l'installation des orpailleurs sur le site.

\section{Impact sur la flore}

Sur la flore et la végétation, il est noté une destruction accélérée du couvert végétal. Le site contenant un champ d'anacarde, il y a eu destruction de cette culture. La coupe des arbres pour le soutènement des puits se fait sur place et sans contrôle. Selon le chef de Cantonnement des Eaux et Forêts de Bouna, "aucun orpailleur ne détient d'autorisation de coupe. Le soutènement utilise en moyenne 15 bois (tronc d'arbre) pour un mètre. Un puits nécessiterait environ un chargement soit environ 400 à 500 troncs d'arbres pour son soutènement. Ce qui fait qu'on assiste à une déforestation de la zone immédiate des deux sites. Par ailleurs, cette destruction du couvert végétal favorise l'érosion et le lessivage ».

L'installation des orpailleurs nécessite le défrichage, la coupe de bois et de pailles pour la construction de maisons ou d'hangars de fortune à usage d'habitation ou commercial.

Quant à la mise en place du puits, elle nécessite également le défrichage, et la coupe de bois pour le soutènement afin d'éviter les éboulements. La savane se trouve ainsi déboisée totalement car aucune surveillance effective n'est mise en place.

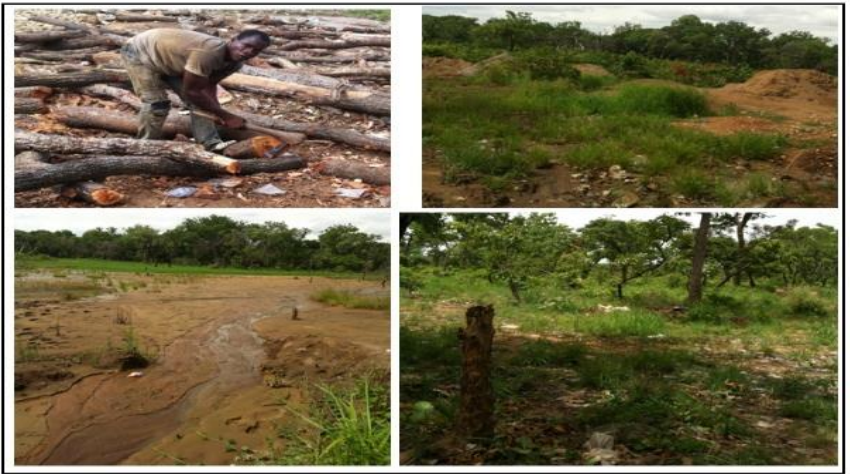

Photo 6: Impacts du fonçage sur la végétation 


\section{Impact sur les ressources en eau}

L'eau intervient dans la réalisation de presque toutes les activités de l'exploitation artisanale de l'or. Lors du fonçage, les orpailleurs atteignent la nappe phréatique qui se situe en moyenne à 14-15 m de profondeur. Ils ont recours à des motopompes pour évacuer des quantités impressionnantes d'eau. Ces motopompes sur le site évacuent chacune plusieurs litres d'eau par jour contribuant ainsi à diminuer le niveau de la nappe d'eau souterraine. Aussi, l'abandon des piles usées à l'intérieur des puits peut-il polluer les ressources en eaux souterraines. Les moulins qui assurent le broyage du minerai utilisent de l'eau pour refroidir le moteur. Même si la consommation d'eau n'est pas significative à ce niveau, il faut noter le déversement des huiles usées et d'hydrocarbures qui peuvent atteindre les ressources en eaux.

Les activités lors des étapes de lavage et d'extraction de l'or par le mercure sont les plus consommatrices d'eaux. A titre d'exemple, il faut environ 200 litres d'eau pour le lavage d'un sac de 50kg de " farine " de minerai lors de l'extraction de l'or. Le mercure utilisé contamine les ressources en eau.

A toutes ces activités s'ajoute la vie quotidienne des orpailleurs qui exige un besoin quotidien en eau (nutrition, lessive, douche, etc.). La production de déchets solides et liquides pollue aussi les ressources en eaux par lessivage ou par infiltration. Cette eau est pompée à l'extérieur et se déverse dans la rivière Bineda, un affluent de la Volta et qui se trouve à moins de $200 \mathrm{~m}$ du site de Lomidouo et plus de 500m du site de Kintan. Cette eau, utilisée par les résidents des deux sites pour les besoins quotidiens (toilette, cuisson des aliments et boisson par certains), peut constituer une source d'intoxication pour les riverains.

En somme, les impacts sur l'eau sont l'épuisement des ressources en eau (utilisation massive d'eau, rejets d'eau lors du fonçage), la pollution des eaux de surfaces et/ou souterraines, la destruction du lit de la rivière Bineda.

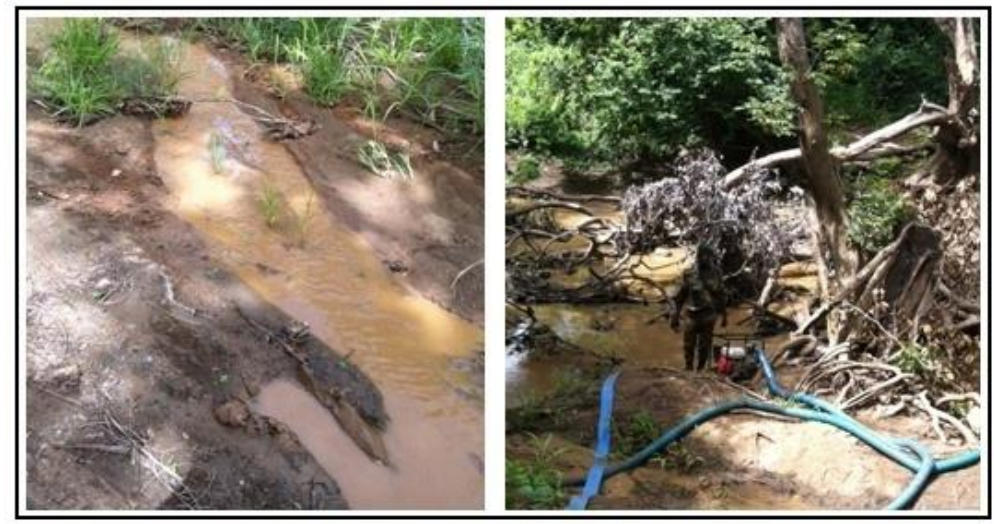

Photo 7: Impacts du fonçage sur les ressources en eau 


\section{Impact sur le sol et insécurité alimentaire}

Le sol est impacté d'une manière ou d'une autre à toutes les étapes de l'exploitation artisanale de l'or. Lors de l'identification du site, de petites galeries sont creusées çà et là ; ce qui contribue à la dégradation du sol par érosion. Il en est de même lors du fonçage durant lequel les puits sont creusés afin d'extraire le minerai. Ce qui dégrade le sol de façon irréversible car les sols ne sont pas reconstitués après abandon des sites, modifiant ainsi le paysage. La pollution visuelle est accentuée par les déchets sur le sol. A cela, il faut ajouter les pollutions du sol engendrées par les déchets solides et liquides produits sur le site (huiles, hydrocarbures, fèces humains, plastique, eaux résiduaires, etc.). Il y a surtout la contamination des sols par les produits chimiques (mercure) utilisés lors de l'extraction de l'or. Les impacts sur le sol sont l'érosion, l'infertilité, la pollution par les déchets solides et liquides, la contamination par des substances nocives. Cette situation est plus perceptible au niveau de la production alimentaire et participe à la modification des habitudes alimentaires. En effet, la dégradation du sol étant un facteur de réduction des surfaces cultivables et aussi un facteur de mobilité à la recherche de terres plus fertiles, elle est la résultante d'une diminution des productions alimentaires et la naissance d'actes conflictuels dans la question d'occupation des terres. Le témoignage de Dame O.K l'illustre bien. "L'activité d'orpaillage qui se pratique actuellement se déroule sur la portion de terre familiale. C'est juste à coté que personnellement je défriche mon champ d'ignames. Aujourd'hui je ne sais plus où cultiver à cause de l'exécution de cette activité aurifère. Il va falloir solliciter de l'espace; ce qui n'est toujours pas évident de l'obtenir, compte tenu de la course à la sécurisation des terres dans notre zone ».

Le fonçage a pour impacts la dégradation du sol qui est laissé au ravinement, l'érosion des sols, la pollution des sols par les piles laissées dans le puits. Des monticules de minerai stérile sont épandus aux alentours des puits impliquant des pertes pour certaines activités (élevage et agriculture). Par ailleurs, les déchets (sachets plastiques, eaux usées, piles usées, vêtements) de l'extraction se répandent un peu partout sur le sol du site. Plus encore, les sols sont pollués par les déversements des huiles de vidange et par les hydrocarbures des pompes. 


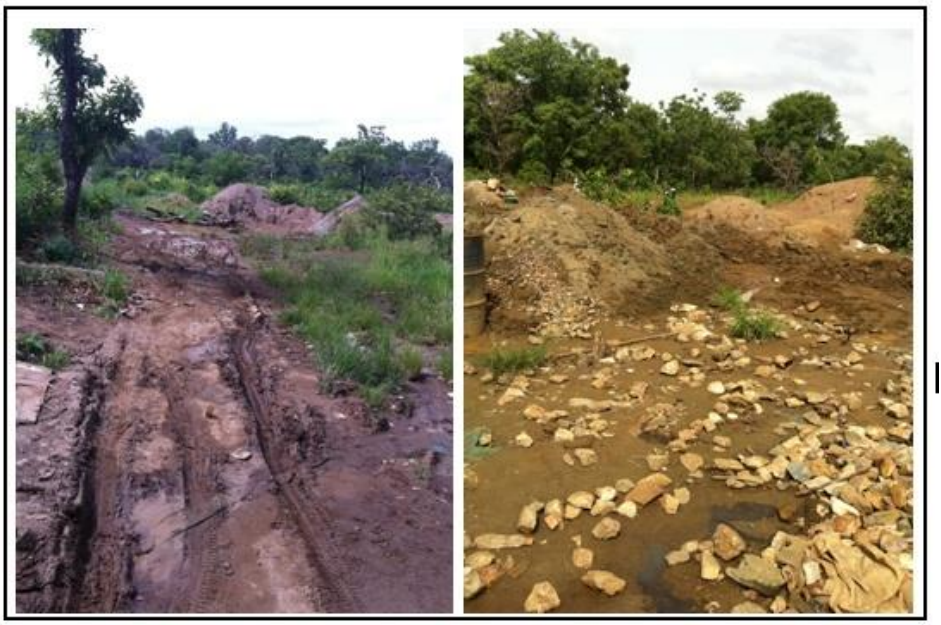

Photo 8: Impacts du fonçage sur le sol

\section{Impact sur l'air}

La poussière sur le site est remarquable. Plusieurs activités sont source de pollution de l'air particulièrement le broyage du minerai. La quantité de poussière produite par le fonçage étant moindre du fait de l'atteinte de la nappe phréatique par les fonceurs. On note également la présence de fortes émissions de gaz, de fumées mais aussi de bruits (moulins, motopompes et moteurs). L'air est également pollué par les vapeurs de mercure pendant le brûlage de l'amalgame. Quant aux odeurs, elles sont dues à la décomposition des déchets solides et liquides générés par les orpailleurs. Ces déchets sont rejetés dans la nature sans aucune mesure d'assainissement. Par manque d'infrastructures, les orpailleurs défèquent dans la nature. Pendant cette phase, l'air est pollué par les poussières particulièrement nocives pour la santé. Les pompes dégagent de fortes quantités de fumées $(\mathrm{CO} 2)$ dans l'air. La nuisance sonore résulte du pompage des eaux souterraines et des outils de fonçage des orpailleurs. Le vent transporte des déchets d'une zone à une autre.

\section{Impact sur la santé}

Les orpailleurs sont le plus souvent exposés à un certain nombre de maladies qui sont liées aux conditions de vie et de travail sur le site. L'infirmier du centre de santé privé installé sur le site de Lomidouo a relevé ceci : "nous assistons à la manifestation de maladies liées au manque d'assainissement sur le site et au manque d'hygiène, mais aussi de nombreux cas d'accidents. Par ordre d'importance, on a le paludisme, les maladies diarrhéiques, les fièvres typhoüdes, le choléra, les dermatoses, etc. L'exposition aux poussières occasionne des maladies respiratoires (toux, pneumonie, Angine, etc.). Les vapeurs de mercure représentent un réel problème de santé publique ». 
. Les risques de transmission des IST/SIDA sont aussi très développés sur le site d'orpaillage par la dépravation des mœurs. La présence des prostituées est signalée sur les sites, mais des entretiens que nous avons eus avec elles, il ressort qu'elles utilisent les méthodes de protection. Le problème réside au niveau des filles commerçantes (le jour) et prostituées de luxe (la nuit) à qui des orpailleurs peuvent donner facilement de grosses sommes quand la production est bonne. A cause de ces sommes, et l'alcool et la drogue aidant, celles-ci peuvent avoir des rapports sexuels avec des orpailleurs sans protection. Changeant sans cesse de partenaires, elles peuvent être source de dissémination des IST/SIDA.

\section{Impact sur l'économie}

L'orpaillage joue un rôle important dans l'économie des orpailleurs et des collectivités locales. Cette activité a surtout un impact très positif sur les emplois. La vente d'un sac d'or peut rapporter jusqu'à 10 millions de francs CFA. L'orpaillage contribue à augmenter les revenus des orpailleurs par la vente directe de l'or et la création des activités annexes qui se développent autour des sites (restauration, bar, boutique, forgeron, vente d'habits, jeux vidéo, etc..). Mais, l'orpaillage occasionne aussi des pertes de terres agricoles et la déforestation, ce qui influe négativement sur l'économie locale.

\section{Impact sur la sécurité}

L'impact de l'orpaillage sur la sécurité est lié aux conditions de travail sur le site et des installations. Le manque d'équipements de protection individuel augmente les risques de blessures. On note sur le site de Kintan une exposition à l'air libre de câbles électriques qui peuvent provoquer des incendies ou des électrocutions mortelles. Le banditisme et l'insécurité sont quasi présents. La présence de nombreux engins à deux roues (charrettes, motocyclettes) occasionne souvent des accidents qui peuvent dans certains cas causer des traumatismes irréversibles.

\section{Chapitre III. Identification Des Risques Encourus Et Mesures D'attenuation Des Impacts}

\section{1-Identification Des Risques Encourus}

La pratique de l'orpaillage possède de nombreux risques aussi bien sur le plan humain que sur l'environnement biophysique.

1-1 Risques sur les populations

\begin{tabular}{|c|c|}
\hline Acteurs & Menaces \\
\hline Creuseurs & $\begin{array}{c}\text { Risques : } \\
\text { d'asphyxie liée au manque d'oxygène dans l'air en } \\
\text { profondeur des galeries ; } \\
\text { d'accidents, de chocs, de chutes de matériaux ; } \\
\text { de prise de stimulants ; } \\
\text { de maladies dues à la mauvaise hygiène ; }\end{array}$ \\
\hline
\end{tabular}




\begin{tabular}{|c|c|}
\hline & $\begin{array}{c}\text { d'intoxication au } \mathrm{CO}_{2} \text { des pompes ; } \\
\text { d'explosion ; } \\
\text { d'absorption cutanée des éléments chimiques constitutifs des } \\
\text { roches }\end{array}$ \\
\hline $\begin{array}{c}\text { Concasseurs et } \\
\text { broyeurs }\end{array}$ & $\begin{array}{c}\text { Risques : } \\
\text { - de maladies liées à l'exposition répétée à la poussière ; } \\
\text { - d'intoxication aux fumées ; } \\
\text { - de blessures par les éclats de pierre dans les yeux; } \\
\text { - d'accidents dûs à la projection du minerai ; } \\
\text { - de maux d'oreilles (surdité). }\end{array}$ \\
\hline $\begin{array}{l}\text { Laveurs au } \\
\text { mercure et } \\
\text { raffineurs }\end{array}$ & $\begin{array}{c}\text { Risques : } \\
\text { de consommation accidentelle du mercure ; } \\
\text { d'intoxication à la vapeur de mercure pouvant entrainer de } \\
\text { troubles comportementaux à long terme ; } \\
\text { de maladie dermatologique liée à la manipulation sans } \\
\text { précautions de produits chimiques dangereux; }\end{array}$ \\
\hline $\begin{array}{l}\text { Populations des } \\
\text { camps de vie }\end{array}$ & $\begin{array}{c}\text { Risques : } \\
\text { de consommation des eaux souterraines et superficielles } \\
\text { polluées par les produits chimiques par les villageois ; } \\
\text { d'incendies dus aux mauvaises installations électriques et } \\
\text { aussi des constructions en pailles ; } \\
\text { d'apparition des maladies vectorielles par la stagnation des } \\
\text { eaux sanitaires des toilettes. }\end{array}$ \\
\hline
\end{tabular}

\section{Risques sur l'environnement biophysique}

Les risques sur l'environnement biophysique sont surtout ceux de pollution et de déforestation. Ils sont liés :

- $\quad$ à la mauvaise utilisation du mercure qui entraine le relâchement des vapeurs dans l'air et liquide dans l'eau;

- $\quad$ au déversement dans la nature des produits toxiques (carburants, des huiles de vidange et de manière systématique des boues de traitement);

- $\quad$ à l'eutrophisation du milieu aquatique résultant de l'augmentation de la concentration en matières en suspension (colorants) des cours d'eau recevant les boues de traitement ;

- $\quad$ au déversement des carburants et des huiles de vidange des motopompes et machines de broyage se retrouvant en permanence sur le site. Mal stockés et volontairement ou inconsciemment abandonnés, ils peuvent polluer les sols et la nappe phréatique.

\section{Mesures d'attenuation Des Impacts}

Ces mesures permettront de réduire les impacts négatifs constatés sur les sites d'orpaillage tant sur le milieu humain que sur le milieu biophysique. Il s'agit de :

- $\quad$ réhabilitation progressive des sites ;

- $\quad$ remblaiement des puits abandonnés ; 
- $\quad$ plan de gestion des déchets

- reboisement ;

- utilisation des barres de fer en remplacement du bois dans le soutènement ;

- $\quad$ laisser intact si possible certain endroit avec une importante ressource faunique ;

- $\quad$ équipement de protection individuel ;

- $\quad$ éloigner les moulins de broyage du comptoir, entretien régulier des appareils, ouvrage de détournement du cours d'eau, ouvrage de drainage des eaux ;

- $\quad$ sensibilisation à l'élaboration d'un plan de gestion des déchets solides et liquides sur les sites ;

- $\quad$ infrastructures et personnel de santé,

- $\quad$ sensibilisation à l'hygiène ;

- dédommagement des populations ;

- $\quad$ création d'activités génératrices de revenus ;

- $\quad$ dédommagement des agriculteurs et éleveurs ;

- $\quad$ mettre sur pied un comité de gestion des conflits entre les populations et les orpailleurs ;

- $\quad$ mise en place d'un système de sécurité et de surveillance.

\section{Discussions}

Cette étude qui a porté sur les activités de l'orpaillage présente des similitudes avec celles réalisées par d'autres auteurs. Kouadio (op.cit.) indique que la pratique de l'or a occasionné des changements dans la structure sociale et économique par son adoption au sein de la population de Hiré. Ces changements sont constatés au niveau de rapports de production économique au sein de l'unité familiale et se manifestent par des contestations de l'autorité ou par des conflits d'autorité entre aînés et cadets sociaux. Par ailleurs, l'utilisation de mercure dans la purification de l'or et aussi certaines particules chimiques contenues dans les résidus de pierres et du sous-sol entraînent des dépôts sédimentaires qui polluent les milieux aquatiques et atmosphériques ; tout comme cela a été démontré dans cette recherche. En Guyane, les recherches effectuées par Polidori et al (2001) portant sur le cycle biochimique du mercure ont mis en évidence le rôle aggravant de l'activité aurifère et particulièrement de l'orpaillage, d'une part par les rejets supplémentaires de mercure métallique ; d'autre part par une érosion certaine des sols qui favorise la mobilisation et le transport du mercure métallique jusqu'aux points les plus bas (bas-fond, cours d'eau). Les résultats de ces auteurs sont confirmés dans la présente étude. Sur la santé humaine, les vapeurs de mercure représentent un réel problème de santé publique pour les orpailleurs et les populations riveraines. Des études relatives à l'utilisation du mercure dans le traitement du 
minerai menées sur 11 sites d'orpaillage au Burkina (Ouédraogo, 2006) illustre bien l'impact de l'exploitation artisanale de l'or sur la santé. La valeur moyenne de mercure trouvée est de 194,5ug/g dans l'urine. 98,9\% ont des concentrations urinaires en mercure au-delà des valeurs de référence de la population générale ; $68,8 \%$ ont des concentrations au-delà des valeurs de référence professionnelles $(35 \mathrm{ug} \mathrm{Hg} / \mathrm{g})$ et près de la moitié $(49,5 \%)$ présente des valeurs supérieures à $100 \mathrm{ug} \mathrm{Hg} / \mathrm{g}$. Dans le "Faso.net", un article paru le 18 septembre 2006, un affrontement a opposé orpailleurs exploitant le site de Fafora au Burkina-Faso et la population autochtone. Les autochtones reprochent aux orpailleurs de pratiquer un déboisement intensif, de leur manquer de respect, de souiller leurs lieux sacrés et enfin de voler leurs volailles. Ils les accusent également de délits d'adultère. Dans la commune rurale de Kompti par exemple, pour le seul mois de Juillet 2006, le service social a été sollicité pour la résolution de cinq (5) cas de viols, six (6) cas de drogues et onze (11) cas de conflits conjugaux. Selon cette même source, les services d'actions sociales et de solidarité nationale de Gaoua et de Kompti ont noté une multiplication des cas de viols, de trafics d'enfants, de conflits de famille du fait de la présence des orpailleurs (Human Rights Watch, 2005). Il faut noter que la dynamique de l'activité aurifère, même si se présentant comme un facteur de croissance économique pour des Etats et qui devrait impacter positivement la vie des populations, contribue au développement de phénomènes néfastes sur la population et la nature en général.

\section{Conclusion}

Au terme de cette étude, il ressort que l'exploitation artisanale de l'or sur les sites de Lomidouo et Kintan montre que l'orpaillage a des impacts au niveau de l'environnement social, la faune, la flore, les ressources en eau, le sol et l'insécurité alimentaire, l'air, la santé, l'économie, etc., dans la Région du Bounkani.

Le cadre méthodologique a eu pour axe de référence scientifique le développement rural lié à la question du changement social et la méthode qualitative a été privilégiée à travers les différents entretiens réalisés avec des acteurs clés. La question centrale étant de rechercher les impacts de l'exploitation artisanale de l'or sur l'environnement naturel et sur les conditions de vie socio-économiques des populations, le structurofonctionnalisme a été retenu comme étant l'une des méthodes la plus appropriée pour permettre d'appréhender les structures et leur fonctionnement afin de comprendre et d'expliquer les différents problèmes socioenvironnementaux des populations.

L'analyse et l'interprétation des données indiquent que l'activité d'orpaillage ancrée dans les habitudes des populations, bien que génératrice de revenus et donc de développement rural, est source de nombreux impacts 
sur l'environnement et sur la santé des populations. Pour ce qui concerne le développement rural, l'activité d'orpaillage a impacté les conditions de vie des acteurs. Ceci se traduit par la construction d'un bâtiment de trois classes pour les écoliers de Kintan et la réalisation d'une pompe villageoise permettant à la population de Lomidouo de s'alimenter en eau potable. En ce qui concerne l'impact environnemental, l'exploitation artisanale de l'or sur ces différents sites est source de dégradation, de pollution, de raréfaction de certaines essences forestières comme le karité, ce qui entraine la perte de la biodiversité.

Face aux réalités dichotomiques (aspect positif et aspect négatif) que présente l'exploitation artisanale de l'or, les acteurs potentiels que sont les orpailleurs, les chefs coutumiers, la population riveraine, sans oublier les décideurs doivent œuvrer pour une bonne organisation de cette activité afin d'atténuer et prévenir les populations sur les incidences néfastes sur l'homme et son environnement, ses avantages ne devant pas conduire à perdre de vue les conséquences graves d'une telle activité. La mise en place d'une plateforme d'innovation pour l'activité aurifère est donc envisageable afin que tous les acteurs concernés par cette activité y compris la population puissent en tirer profit et sauvegarder l'environnement. Ceci est le gage d'un développement durable du secteur aurifère.

\section{References:}

ORCADE (2006). Etude diagnostique du cadre institutionnel et juridique de l'activité minière industrielle au Burkina Faso : cas de Poura et Essakane. Rapport d'étude.

OUEDRAOGO B, H. (2006). L'impact de l'exploitation artisanale de l'or (orpaillage) sur la santé et l'environnement. Gestion des substances toxiques, Portail Afrique de l'Ouest, http://www.mediaterre.org/afrique-ouest/actu,20061121095625.html.

MINISTERE DES MINES DE L'ENERGIE ET DU PETROLE (2013). Code minier ivoirien

NDELA KUBOKOSO, J (2008). Les activités minières et la fiscalité : Cas de la République Démocratique du Congo. Thèse de doctorat en Droit, Administration et Secteur Public. Université Paris I Panthéon - Sorbonne. POLIDORI L., FOTSING J.-M., ORRU J.- F., 2001. Déforestation et orpaillage : apport de la télédétection pour la surveillance de l'occupation du sol en Guyane française. In : Carmouze J.-P., Lucotte M., Boudou A. (éd.). Le mercure en Amazonie. Paris, France, Ird éditions, p. 473-494.

KOUADIO KOUASI, N (2003). Exploitation artisanale de l'or dans le processus de mutation socio- économique à Hire (Sud Bandama Côte d'Ivoire), D.E.A, Université de Bouaké (Côte d'Ivoire), Bouaké. HUMAN RIGHTS WATCH (2005). Le Fléau de l'or. Rapport. Kinshasa. 
JAQUES E. et ZIDA, B (2004). La filière artisanale de l'or au Burkina Faso : bilan, perspectives d'évolution et recherche de cibles pour le développement de petites mines. MAE/CIFEG regional workshop, Ouagadougou, Burkina Faso - November 2003. CIFEG Occasional Publication 2004/39, pp. 41- 59.

\section{Sites visités}

www.un.org/french/events/wssd/pages/cnued.htm

http://www.avmaroc.com/actualite/orpaillage-burkina-a137229.html 